\title{
Nonlinear interactions of nearly non-dispersive equatorial shallow-water waves
}

\author{
Mian WaNg AND ZhaN WANG* \\ Key Laboratory for Mechanics in Fluid Solid Coupling Systems, Institute of Mechanics, Chinese \\ Academy of Sciences, Beijing 100190, China \\ *Corresponding author: zwang@imech.ac.cn \\ AND \\ HENNES HAJDUK \\ Institute of Applied Mathematics (LS III), TU Dortmund University, Vogelpothsweg 87, D-44227 \\ Dortmund, Germany hennes.hajduk@math.tu-dortmund.de
}

[Received on 1 August 2019; revised on 2 March 2020; accepted on 3 March 2020]

\begin{abstract}
This paper is concerned with nonlinear interactions of fundamental equatorial modes. In order to understand the mechanism of large-scale atmospheric motions in the near equator regime-especially the observed wavenumber-frequency spectrum-we develop novel models describing interactions among Kelvin, Yanai and Poincaré waves. Based on the methods of multiple scales and Galerkin projection, the primitive equations can be simplified to model equations which reduce the complexity and cost of computation significantly. Subsequently, the detailed numerical results indicate that wave interactions between the aforementioned modes in the non-dispersive regime depends on initial amplitude and relative phase and that the eastward Yanai wave can be generated from the second Poincaré mode. We also compare the simplified models to an advanced finite element approximation for the primitive equations. The fact that results of the latter are in good agreement, at least qualitatively, with those of the simplified models, indicates that reduced models capture most of the wave interaction mechanisms in the nearly non-dispersive regime.
\end{abstract}

Keywords: wave interactions, shallow water equations, equatorial waves.

\section{Introduction}

Equatorial waves trapped in a waveguide due to the singularity of the equator where the Coriolis parameter vanishes play an important role in many aspects of tropical weather and climate. For example, in the atmosphere, equatorial waves have been tentatively associated with the mesoscale variability of convection in the tropics (Takayabu, 1994), whereas their propagation in the stratosphere is the key element of the stratospheric quasi-biennial oscillation (Baldwin et al., 2001).

There have been plenty of studies in regard to equatorial waves. In the 1960s, the linear theory of equatorially trapped waves was put forward by Matsuno (1966) and Lindzen (1967), who showed that solutions to the linearized equatorial shallow water (ESW) equations employing the $\beta$-plane approximation can be classified into two categories: a non-dispersive Kelvin wave propagating only in the eastward direction, and other dispersive waves including Yanai (mixed Rossby-gravity), Rossby and Poincaré (inertia-gravity) modes. Wallace (1973) first showed the observation of equatorial Kelvin waves in the lower stratosphere and pointed out its connection with the quasi-biennial oscillation. Yanai \& Maruyama (1966) found observational evidence of the signal of motions which was similar 
to Yanai waves in the equatorial region, and then Maruyama (1967) documented fluctuations in the meridional wind in the stratosphere associated with these particular waves. Based on the work of Takayabu (1994), Wheeler \& Kiladis (1999) calculated a global space-time spectrum of a long record of tropical cloudiness. The spectrum was found for the equatorial atmospheric variability, giving further evidence that some of the spectral peaks correspond to particular equatorial wave modes. By dealing with expanded dataset, they found similar phenomenon that prominent spectral peaks are oriented along the dispersion curves of the ESW equations and there are prominent Kelvin waves and Yanai waves generating in the structure of equatorial atmosphere.

In order to deeply understand the equatorial motions, the mechanism of long-time development of equatorial waves and their nonlinear interactions need to be elucidated. To assess the importance of nonlinearity for long-time self-interactions of Kelvin waves, Boyd (1980) and Ripa (1982) independently derived the inviscid Burgers equation which controls the evolution of the interaction in the weakly nonlinear regime. Special resonances among different equatorial modes were subsequently considered by Boyd, including long-short wave interactions for Rossby-Rossby or Rossby-Poincaré modes (Boyd, 1983a) and second harmonic resonance between two Rossby waves or two westward-travelling Poincaré waves (Boyd, 1983b). Almost at the same time, Ripa (1983a,b) investigated resonant triads among different harmonic modes for general cases. For resonant triads, the wave with the highest absolute frequency always acts as an energy source (or sink) for the remaining triad components (Marcus, 2008). For resonantly interacting equatorial waves, high-frequency modes are found to be energetically more active than low-frequency modes (Raupp \& Dias, 2010).

In addition, the understanding of the excitation of Yanai waves is also very important because of the observations of equatorial instability waves (which feature the characteristics of Yanai waves) and their role in the momentum and heat budgets in the tropics (Yoshikazu, 1970). There exist several theoretical mechanisms proposed by different groups to explain the generation and propagation of Yanai waves. Based on the multi-scale analysis Majda et al. (1999) proposed, asymptotic models to investigate the coupling of Kelvin waves with Yanai or Rossby waves through topography resonances showing that the Kelvin wave can excite large-scale Yanai and Rossby waves through topographic resonances. A nonlinear wave-conditional instability of the second kind mechanism, which was carefully examined by Itoh \& Ghil (1988), can also produce Yanai waves through the unstable interaction between equatorial waves and cumulus convection. Kelly et al. (1995) found that Yanai waves can be generated by a simple cross-equatorial wind forcing. Using a spectral method Raupp \& Dias (2005) showed that Yanai waves are excited by a nonlinear mechanism in which the slow modes excited by the thermal forcing generate a quasi-geostrophic basic state that supplies energy especially to the mixed Rossby-gravity waves with zonal wavenumber 4-5 and the period of 4-5 days.

In this paper, we attempt to extend the previous asymptotic studies by deriving new reduced model equations using the method of multiple scales to investigate the Kelvin-Yanai-Poincaré interactions and the energy exchanges among these modes. Field observations presented by Wheeler \& Kiladis (1999) and Kiladis et al. (2009) show a strong peak band in intermediate-scale Kelvin and eastward propagating Yanai waves (see Fig. 1 in Kiladis et al., 2009). Motivated by their work, we focus on eastward-propagating equatorial waves with large zonal wavenumbers. The key observation is that for considerably large wavenumbers, the phase speeds of Yanai and Poincaré waves approach the speed of the Kelvin wave (Fig. 1). For example, for zonal wavenumber $k=3$, the speed difference between the Kelvin wave and the eastward propagating Yanai wave is less than $10 \%$. This fact implies that these modes are almost non-dispersive in this circumstance therefore, nonlinear interactions among these basic modes can take place in the long-time dynamics. Based on triads or quartets, we propose a 


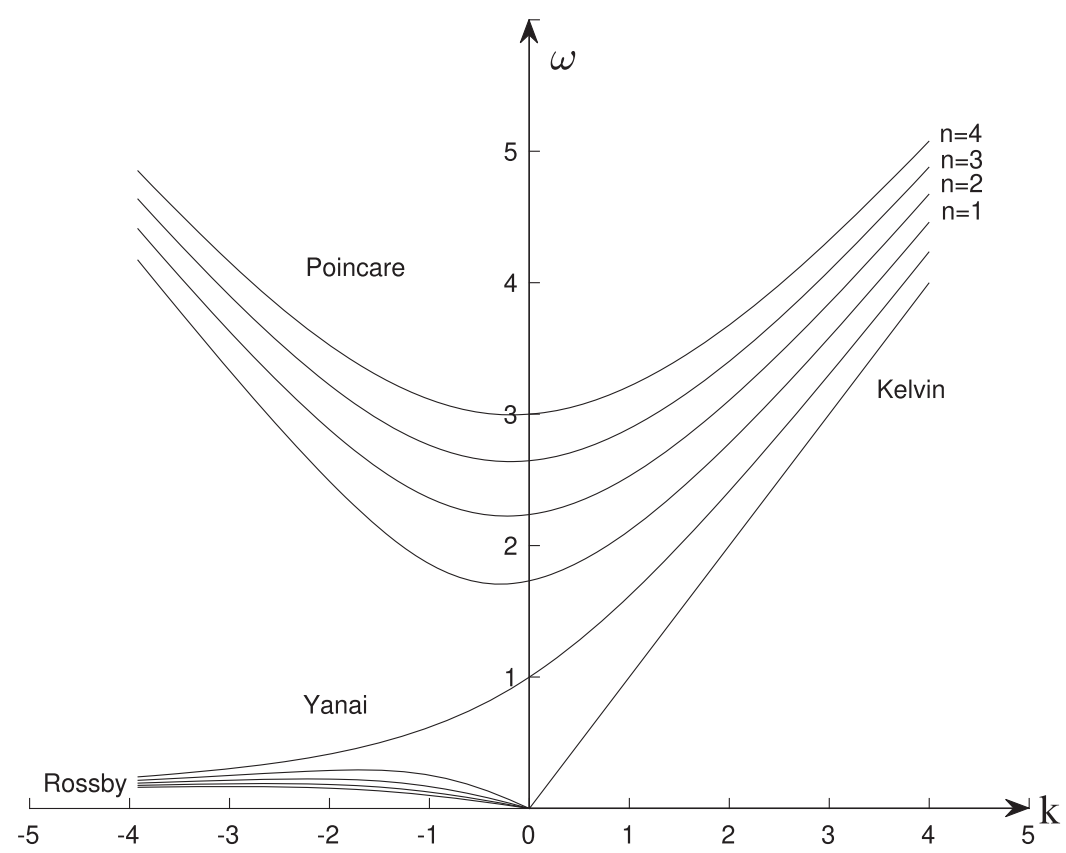

FIG. 1. Dispersion relation for equatorial waves with curves corresponding to the Kelvin, Yanai, Rossby and Poincaré waves.

theoretical mechanism through which Yanai waves can be excited by other equatorial modes, and energy can be transferred among different modes through interactions.

The rest of the paper is organized as follows. The reduced model equations are derived in Section 2, using asymptotic expansion and Galerkin projection. In the same section, the mechanism for exciting intermediate-scale Yanai waves due to nonlinear wave interactions is elucidated by numerical simulations of the obtained models. In Section 3, an improved discontinuous Galerkin method, which can guarantee high-order accuracy, is used to solve the primitive equations and numerical experiments are carried out to validate the newly developed models. Finally, we give concluding remarks in Section 4.

\section{Model equations}

\subsection{Basic equations, non-dimensionalization and linear waves}

Rotation and density stratification are two key factors for large-scale motions of geophysical fluids. In the classical shallow water theory, the fluid is assumed to be a thin homogeneous layer which excludes the vertical structure. Therefore, the buoyancy effect is neglected in the ESW equations and two remaining restoring forces are gravity and Coriolis force. On the other hand, since we focus on nonlinear wave interactions among different equatorial modes, we neglect boundary effect, external forcing and dissipation (such as topography, evaporation-wind feedback and diabatic heating in moist processes) for simplicity, though they may play significant roles in climate and weather in tropics. The ESW equations can be obtained by taking depth-averaged variables as unknowns and by making use of 
the $\beta$-plane approximation. The dimensional ESW equations in the equatorial waveguide are given by

$$
\left\{\begin{array}{l}
\eta_{t}+[(H+\eta) u]_{x}+[(H+\eta) v]_{y}=0 \\
u_{t}+u u_{x}+v u_{y}+g \eta x-\beta y v=0 \\
v_{t}+u v_{x}+v v_{y}+g \eta_{y}+\beta y u=0
\end{array}\right.
$$

where $u$ and $v$ are horizontal zonal and meridional velocity components, respectively, $g=9.8 \mathrm{~m} / \mathrm{s}^{2}$ is the acceleration due to gravity, $H$ is the equivalent depth of the fluid layer, $\eta$ is the surface displacement from the equilibrium position and $\beta$ is a constant called the Coriolis parameter. In practice, the equivalent depth is in the range of 12-50 m (see Wheeler \& Kiladis, 1999); therefore, the reference speed of shallow-water waves $\sqrt{g H}$ is about $11-22 \mathrm{~m} / \mathrm{s}$, which is similar to the typical speed of Kelvin waves (about $10-15 \mathrm{~m} / \mathrm{s}$ ). $\beta=2 \Omega / R \approx 2 \times 10^{-11} / \mathrm{m} / \mathrm{s}$, where $\Omega=2 \pi /$ day and $R \approx 6371 \mathrm{~km}$ is the radius of the earth. We non-dimensionalize the system by choosing

$$
H,[g H]^{1 / 2},\left[\frac{g H}{\beta^{2}}\right]^{1 / 4},\left[\frac{1}{\beta^{2} g H}\right]^{1 / 4}
$$

as the amplitude, velocity, length and time scales, respectively. Therefore, after rescaling, we can remove the constants $g$ and $\beta$ from the system, which now reads

$$
\left\{\begin{array}{l}
\eta_{t}+[(1+\eta) u]_{x}+[(1+\eta) v]_{y}=0 \\
u_{t}+u u_{x}+v u_{y}+\eta_{x}-y v=0 \\
v_{t}+u v_{x}+v v_{y}+\eta_{y}+y u=0
\end{array}\right.
$$

The first step towards understanding the tropical dynamics is the study of small-amplitude motions, which allows a linearization of the primitive equations to identify fundamental modes of oscillation of the system. The linearization of system (2.3) was initially solved by Matsuno (1966), and the solutions can be divided into two categories: a non-dispersive Kelvin wave and countably many dispersive modes. The Kelvin wave takes the form

$$
\left\{\begin{array}{l}
\eta=e^{-\frac{y^{2}}{2}} K(x-t) \\
u=e^{-\frac{y^{2}}{2}} K(x-t) \\
v=0
\end{array}\right.
$$

where $K$ is an arbitrary function, and the dispersive solutions to the linearized equations are listed as follows without a detailed solving process:

$$
\left\{\begin{array}{l}
\eta=\left[\frac{i y H_{n}(y)}{\omega-k}-\frac{i \omega H_{n}^{\prime}(y)}{\omega^{2}-k^{2}}\right] e^{-\frac{y^{2}}{2}} e^{i(k x-\omega t)}, \\
u=\left[\frac{i y H_{n}(y)}{\omega-k}-\frac{i \omega H_{n}^{\prime}(y)}{\omega^{2}-k^{2}}\right] e^{-\frac{y^{2}}{2}} e^{i(k x-\omega t)}, \\
v=H_{n}(y) e^{-\frac{y^{2}}{2}} e^{i(k x-\omega t)}
\end{array}\right.
$$


where $H_{n}(y)$ stands for the Hermite polynomial of order $n$, and

$$
\omega^{2}-k^{2}-\frac{k}{\omega}=2 n+1
$$

is the dispersion relation which gives the relation between zonal wavenumber $k$ and frequency $\omega$ (Fig. 1).

As shown in Fig. 1, for considerably large wavenumbers, the phase speeds for Kelvin, eastwardpropagating Yanai $(n=0)$ and eastward-propagating Poincaré $(n \geqslant 1)$ waves are very close; hence, they can stay together for a long time and nonlinear interactions may take place. Since in the current paper the primary focus is short waves where $k$ and $\omega$ are both large, we can rescale the system by choosing $t=\delta \tilde{t}, x=\delta \widetilde{x}$ and $v=\delta \widetilde{v}$ as new variables, where $\delta$ is a small parameter. Substituting the variable transforms into (2.3) and dropping tildes, the system is recast to

$$
\begin{gathered}
\eta_{t}+[(1+\eta) u]_{x}+\delta^{2}[(1+\eta) v]_{y}=0, \\
u_{t}+u u_{x}+\eta_{x}+\delta^{2}\left(v u_{y}-y v\right)=0, \\
v_{t}+u v_{x}+\eta_{y}+y u+\delta^{2} v v_{y}=0 .
\end{gathered}
$$

In the subsequent analyses, the problem can be simplified by neglecting terms with $\delta^{2}$ due to our particular focus.

\subsection{Interaction among Kelvin, Yanai and first Poincaré modes}

In order to study nonlinear interactions between different equatorial modes, we use the multi-scale asymptotic analysis for the ansatz involving modes that we are interested in. In the weakly nonlinear theory, nonlinear behaviours can be observed through the long-time dynamics of the system; therefore, a long-term variable $\tau=\epsilon t=O(1)$ needs to be introduced in the ansatz. For interactions between Kelvin, Yanai and first Poincaré waves, we assume that the solution takes the form of

$$
\begin{aligned}
& \eta=\epsilon e^{-y^{2} / 2}\left[K+y Y+\left(y^{2}-\frac{1}{2}\right) P_{1}\right](\theta, \tau)+\epsilon^{2} \eta_{2}+\cdots, \\
& u=\epsilon e^{-y^{2} / 2}\left[K+y Y+\left(y^{2}-\frac{1}{2}\right) P_{1}\right](\theta, \tau)+\epsilon^{2} u_{2}+\cdots, \\
& v=\epsilon e^{-y^{2} / 2}\left[\left(Z+y Q_{1}\right)(\theta, \tau)+\left(C_{1}+y C_{2}\right)(x, \tau)\right]+\epsilon^{2} v_{2}+\cdots,
\end{aligned}
$$

with

$$
Z_{\theta}=Y, \quad\left(Q_{1}\right)_{\theta}=2 P_{1},
$$

where $\theta=x-t, C_{i}(i=1,2)$ are arbitrary functions determined by the initial condition, and $K, Y$ and $P_{1}$ stand for the amplitudes of Kelvin, Yanai and first Poincaré waves which propagate eastward with the unit speed and vary slowly with $\tau$.

To elucidate the underlying motivation for choosing this ansatz, we take the first Poincaré wave as an example. Motivated by (2.5), we assume that the solution to the linearization of system (2.7) without 
$\delta$ terms can be expressed as

$$
\eta=u=A(x, t)\left(y^{2}-\frac{1}{2}\right) e^{-y^{2} / 2}, \quad v=B(x, t) y e^{-y^{2} / 2},
$$

where functions $A$ and $B$ are to be determined. Substituting these expressions into (2.7) and neglecting nonlinear terms and $\delta$-related terms yield

$$
A_{t}+A_{x}=0, \quad B_{t}+2 A=0 .
$$

Therefore, solving for $A$ and $B$ gives

$$
A=P_{1}(\theta), \quad B=Q_{1}(\theta)+C_{2}(x),
$$

where $P_{1}$ and $C_{2}$ are arbitrary functions and $\left(Q_{1}\right)_{\theta}=2 P_{1}$. It is worth mentioning that the mean over space of $A$ needs to be zero to avoid unbounded growth of $B$ and therefore we can take the mean of $Q_{1}$ to be zero by definition. In the same vein, it is not difficult to verify that $Z_{\theta}=Y$, and both $Z$ and $Y$ have zero mean.

Next, substituting the ansatz into the nonlinear system (2.7), dropping terms with $\delta$ and collecting terms with the second order of $\epsilon$ yield

$$
\begin{gathered}
\left(\eta_{2}\right)_{t}+\left(u_{2}\right)_{x}=-\partial_{\tau}\left[K+y Y+\left(y^{2}-\frac{1}{2}\right) P_{1}\right] e^{-\frac{y^{2}}{2}}-\partial_{\theta}\left[K+y Y+\left(y^{2}-\frac{1}{2}\right) P_{1}\right]^{2} e^{-y^{2}}, \\
\left(u_{2}\right)_{t}+\left(\eta_{2}\right)_{x}=-\partial_{\tau}\left[K+y Y+\left(y^{2}-\frac{1}{2}\right) P_{1}\right] e^{-\frac{y^{2}}{2}}-\frac{1}{2} \partial_{\theta}\left[K+y Y+\left(y^{2}-\frac{1}{2}\right) P_{1}\right] e^{-y^{2}}, \\
\left(v_{2}\right)_{t}+\left(\eta_{2}\right)_{y}+y u_{2}=-\left(Z+y Q_{1}+C_{1}+y C_{2}\right)_{\tau} e^{-\frac{y^{2}}{2}} \\
-\left[K+y Y+\left(y^{2}-\frac{1}{2}\right) P_{1}\right]\left[\left(Z+y Q_{1}\right)_{\theta}+\left(C_{1}+y C_{2}\right)_{x}\right] e^{-y^{2}} .
\end{gathered}
$$

We integrate $((2.8)+(2.9)) \times e^{-\frac{y^{2}}{2}}$ with respect to $y$ over the whole real line. Then, the compatibility condition (namely to avoid unbounded growth of the solution) gives

$$
K_{\tau}+\sqrt{\frac{2}{3}}\left(\frac{3 K^{2}}{4}+\frac{Y^{2}}{4}+\frac{3 P_{1}^{2}}{16}-\frac{K P_{1}}{4}\right)_{\theta}=0
$$


Similarly, integrating $((2.8)+(2.9)) \times y e^{-\frac{y^{2}}{2}}$ with respect to $y$ over the real line yields

$$
Y_{\tau}+\sqrt{\frac{2}{3}}\left(K Y+\frac{Y P_{1}}{2}\right)_{\theta}=0 .
$$

Finally, integrating $((2.8)+(2.9)) \times\left(y^{2}-\frac{1}{2}\right) e^{-\frac{y^{2}}{2}}$ gives

$$
P_{\tau}+\sqrt{\frac{2}{3}}\left(-\frac{K^{2}}{4}+\frac{Y^{2}}{4}+\frac{13 P_{1}^{2}}{48}+\frac{3 K P_{1}}{4}\right)_{\theta}=0 .
$$

Combining the above formulas, we can obtain a complete system for $K, Y$ and $P_{1}$

$$
\left\{\begin{array}{c}
K_{\tau}+\sqrt{\frac{2}{3}}\left(\frac{3 K^{2}}{4}+\frac{Y^{2}}{4}+\frac{3 P_{1}^{2}}{16}-\frac{K P_{1}}{4}\right)_{\theta}=0, \\
Y_{\tau}+\sqrt{\frac{2}{3}}\left(K Y+\frac{Y P_{1}}{2}\right)_{\theta}=0, \\
\left(P_{1}\right)_{\tau}+\sqrt{\frac{2}{3}}\left(-\frac{K^{2}}{4}+\frac{Y^{2}}{4}+\frac{13 P_{1}^{2}}{48}+\frac{3 K P_{1}}{4}\right)_{\theta}=0 .
\end{array}\right.
$$

Equation (2.10) actually implies that $C_{1}$ and $C_{2}$ do not play a dynamic role and therefore can be decoupled from system (2.11). We take $C_{1}$ as an example. Integrating $(2.10) \times e^{-\frac{y^{2}}{2}}$ yields

$$
\begin{aligned}
\left(\int_{-\infty}^{\infty} v e^{-\frac{y^{2}}{2}} \mathrm{~d} y\right)_{t}= & -\int_{-\infty}^{\infty}\left(Z+C_{1}\right)_{\tau} e^{-y^{2}} \mathrm{~d} y \\
& -\int_{-\infty}^{\infty}\left[K Y+K\left(C_{1}\right)_{x}+\left(3 y^{2}-\frac{1}{2}\right) Y P_{1}+y^{2} Y\left(C_{2}\right)_{x}+\left(y^{2}-\frac{1}{2}\right) P_{1}\left(C_{1}\right)_{x}\right] e^{-\frac{3}{2} y^{2}} \mathrm{~d} y .
\end{aligned}
$$

Apparently, the right-hand side of the above equation should be forced to zero (since it is independent of $t$ ); otherwise, $v$ will exhibit a linear growth in $t$. Therefore, taking the mean over $\theta$ and upon noting $\bar{Z}=\bar{Y}=\bar{Q}=\overline{P_{1}}=0$, we can obtain

$$
\left(C_{1}\right)_{\tau}+\sqrt{\frac{2}{3}} \bar{K}\left(C_{1}\right)_{x}+\sqrt{\frac{2}{3}}\left[\overline{K Y}+\frac{1}{2} \overline{Y P_{1}}\right]=0,
$$

where over-bar stands for the mean. $C_{1}$ satisfies a transport equation determined by Kelvin, Yanai and Poincaré waves. Similarly, the governing equation for $C_{2}$ can be derived by integrating $(2.10) \times y e^{-\frac{y^{2}}{2}}$, which takes the form of

$$
\left(C_{2}\right)_{\tau}+\frac{1}{3} \sqrt{\frac{2}{3}} \bar{K}\left(C_{2}\right)_{x}+\frac{1}{3} \sqrt{\frac{2}{3}}\left[2 \overline{K P_{1}}+\overline{Y^{2}}+\overline{P_{1}^{2}}\right]=0 .
$$

Equation (2.11) forms a quasi-linear hyperbolic system of conservation laws. For this type of equations, the local well-posedness of the initial value problem and formations of singular structures 
(including shocks waves, rarefaction waves and contact discontinuity) in finite time were well established. However, the dynamics of nonlinear hyperbolic systems is still of great interest. For example, when we consider the interactions only between Kelvin and Yanai waves, equation (2.11) reduces to

$$
\left\{\begin{array}{l}
K_{\tau}+\sqrt{\frac{2}{3}}\left(\frac{3 K^{2}}{4}+\frac{Y^{2}}{4}\right)_{\theta}=0, \\
Y_{\tau}+\sqrt{\frac{2}{3}}(K Y)_{\theta}=0 .
\end{array}\right.
$$

Initially, if $K(\theta, 0) \neq 0$ and $Y(\theta, 0)=0$, then only the Burgers equation for the Kelvin mode is in play in the whole system before the shock formation. However, once a shock wave appears, the second equation of (2.12) simultaneously becomes efficient to evoke the Yanai mode due to the delta-type singularity of $K_{\theta}$. Though it is beyond the scope of this paper, the generation mechanism and quantitative properties of delta shocks merit a careful investigation.

Another example can be directly shown by rearranging system (2.11). Adding and subtracting the first two equations of (2.11) yield

$$
(K \pm Y)_{\tau}+\sqrt{\frac{2}{3}}\left[\frac{3 P_{1}^{2}}{16}-\frac{K P_{1}}{4} \pm \frac{Y P_{1}}{2}+\frac{1}{4}(3 K \pm Y)(K \pm Y)\right]_{\theta}=0,
$$

and the third equation of (2.11) can be rewritten as

$$
\left(P_{1}\right)_{\tau}+\sqrt{\frac{2}{3}}\left[\frac{3 K P_{1}}{4}+\frac{13 P_{1}^{2}}{48}-\frac{1}{4}(K-Y)(K+Y)\right]_{\theta}=0 .
$$

It can be deduced from (2.13) and (2.14) that if initially $P_{1}(0, \theta)=(K \pm Y)(0, \theta)=0$, then $P_{1}$ and $K \pm Y$ will stay zero forever (but $K$ and $Y$ are not necessarily zero). This observation indicates that the first Poincaré mode may not be evoked by Kelvin and Yanai waves under certain initial conditions.

Nonlinear interactions between the Kelvin and Yanai waves in the non-dispersive regime were first considered by Milewski and his collaborators (personal communications). Furthermore, they extended equation (2.12) to involve a slight dispersive effect by setting $\delta^{2}=\epsilon$, and the obtained system is of the form

$$
\left\{\begin{array}{l}
K_{\tau}+\sqrt{\frac{2}{3}}\left(\frac{3 K^{2}}{4}+\frac{Y^{2}}{4}\right)_{\theta}=0, \\
Y_{\tau}+\sqrt{\frac{2}{3}}(K Y)_{\theta}-\int_{0}^{\theta} Y(\xi, \tau) \mathrm{d} \xi+\frac{1}{\lambda} \int_{0}^{\lambda} \int_{0}^{\theta} Y(\xi, \tau) \mathrm{d} \xi \mathrm{d} \theta=0,
\end{array}\right.
$$

where $\lambda$ is the period in the $\theta$ direction. We omit the detailed derivations but remark that similar dispersive effects can also be obtained in the presence of Poincaré modes under the same scaling assumption.

Next, we investigate nonlinear wave interactions by numerically solving system (2.11). The new model (2.11) reduces a two-spatial-dimensional problem to a one-dimensional one. More importantly, in contrast to the primitive equations, (2.11) is a typical hyperbolic system of conservation laws, since the non-dispersive part of each fundamental equatorial mode is extracted and combined together. There 
(a) $\tau=0$

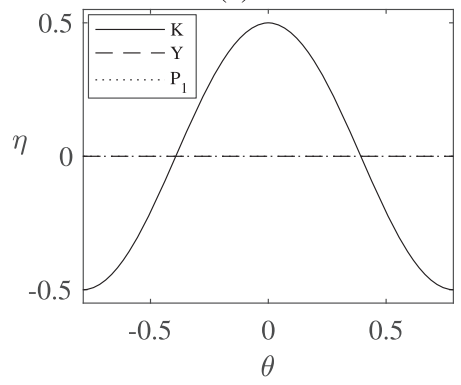

(b) $\tau=2$

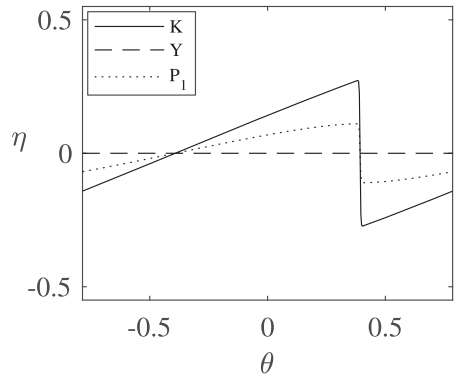

(c) $\tau=5$

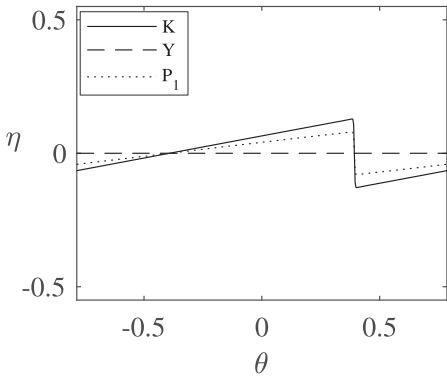

FIG. 2. Nonlinear wave interactions among Kelvin (solid line), Yanai (dashed line) and first Poincaré (dotted line) modes. The initial condition is given by $K=0.5 \cos (4 \theta)$ and $Y=P_{1}=0$, which is shown in (a). Snapshots are taken at (b) $\tau=2$ and (c) $\tau=5$.

(a) $\tau=0$

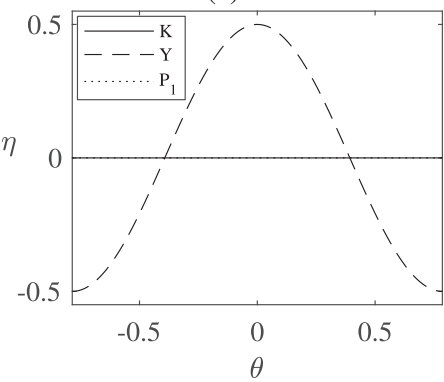

(b) $\tau=2$

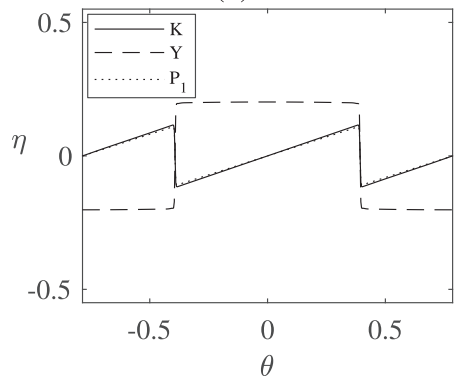

(c) $\tau=5$

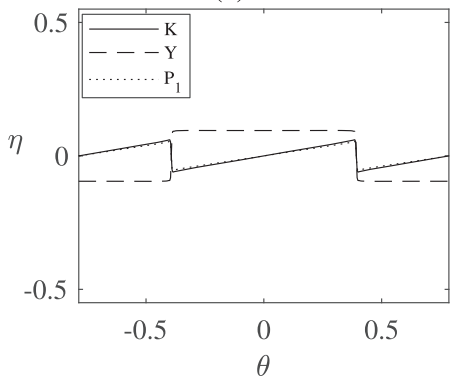

FIG. 3. Nonlinear wave interactions among Kelvin (solid line), Yanai (dashed line) and first Poincaré (dotted line) modes. The initial condition is given by $P_{1}=0.5 \cos (4 \theta)$ and $K=Y=0$, which is shown in (a). Snapshots are taken at (b) $\tau=2$ and (c) $\tau=5$.

are many excellent numerical schemes developed in the past few decades for conservation laws. In the current paper, the fifth-order WENO scheme, a mature, high-resolution and shock-capturing algorithm, together with the fourth-order explicit Runge-Kutta method for time integration, is used to solve (2.11). In what follows, all the numerical experiments were carried out for the wavenumber $k=4$.

Figures 2- 4 show how energy transfers from one mode to the other two. When there is only a Kelvin (or Poincaré) wave initially, it transfers energy to the Poincaré (or Kelvin) wave, but no Yanai wave emerges. Shock waves appear in both Kelvin and Poincaré waves during the interaction. For the initial data consisting of a single Yanai wave, both Kelvin and first Poincaré modes can be wakened. After a period of time, all these waves develop shocks. It is noted that the generated Kelvin and Poincaré waves are locked in phase and the amplitudes are approximately equal. They exhibit twice the wavenumber of the Yanai wave. Comparing Fig. 4 with Fig. 2, it is found that the energy transferred from the first Poincare mode to the Kelvin wave is close to that of the inverse process in its early development. The numerical experiments were conducted in the domain $[-\pi, \pi]$ using a uniform meshgrid with $\Delta \theta=\frac{\pi}{1000}$, and only one period is shown in figures.

Figures 5- 9 show how two types of waves interact with each other. It is shown in Fig. 5 that when there are in-phase Kelvin and Yanai waves of the same amplitude initially, they preserve the in-phase property during the whole process and no Poincaré waves are excited. For an out-of-phase 
(a) $\tau=0$

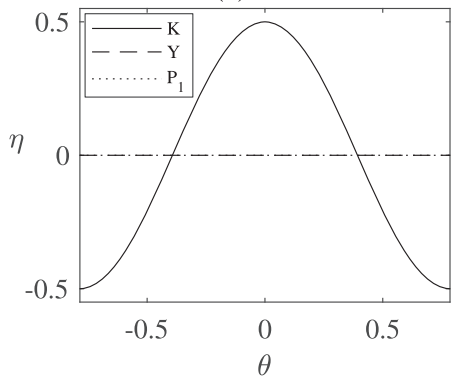

(b) $\tau=2$

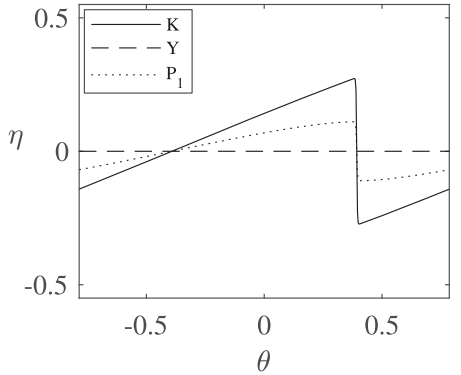

(c) $\tau=5$

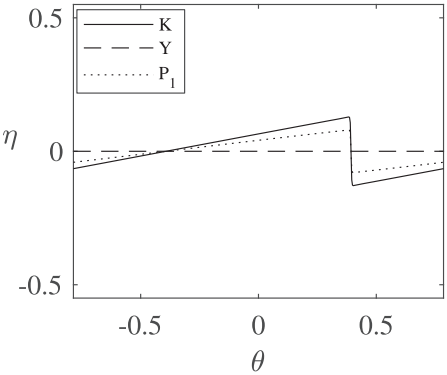

FIG. 4. Nonlinear wave interactions among Kelvin (solid line), Yanai (dashed line) and first Poincaré (dotted line) modes. The initial condition is given by $Y=0.5 \cos (4 \theta)$ and $K=P_{1}=0$, which is shown in (a). Snapshots are taken at (b) $\tau=2$ and (c) $\tau=5$.

(a) $\tau=0$

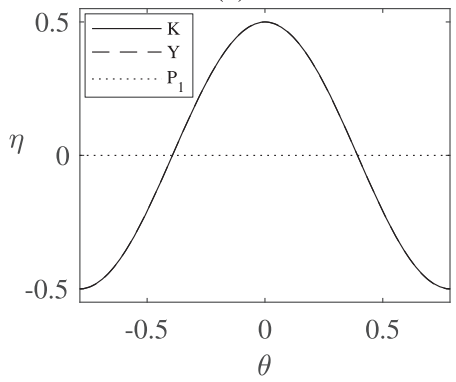

(b) $\tau=2$

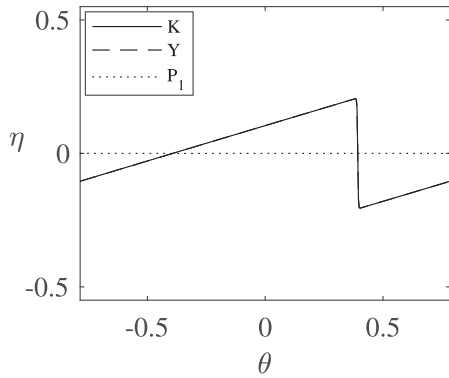

(c) $\tau=5$

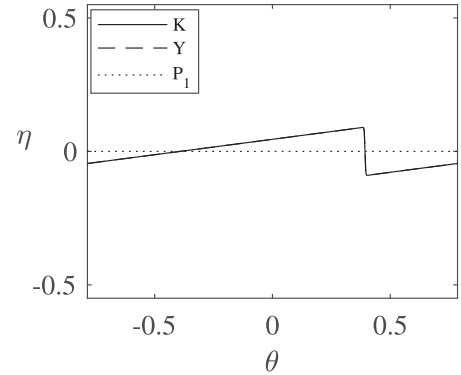

FIG. 5. Nonlinear wave interactions among Kelvin (solid line), Yanai (dashed line) and first Poincaré (dotted line) modes. Two in-phase waves exist in the initial condition: $K=0.5 \cos (4 \theta), Y=0.5 \cos (4 \theta)$ and $P_{1}=0$, which are shown in (a). Snapshots are taken at (b) $\tau=2$ and (c) $\tau=5$.

(a) $\tau=0$

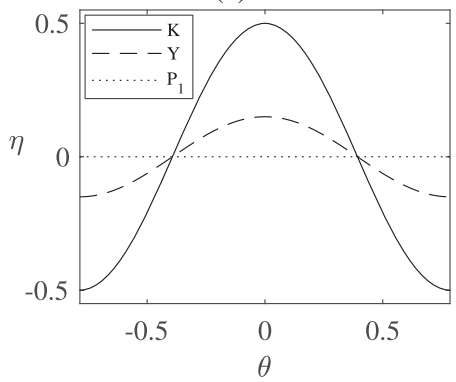

(b) $\tau=2$

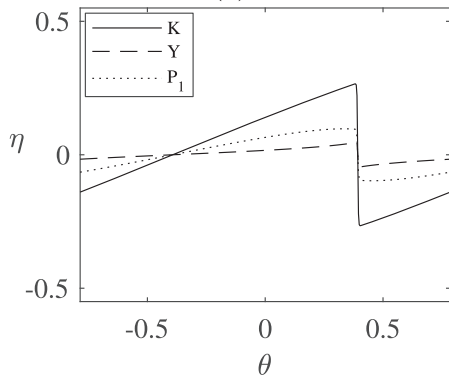

(c) $\tau=5$

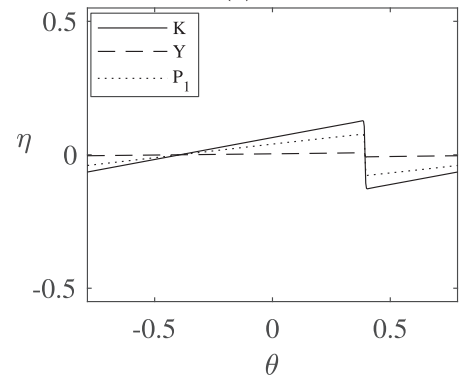

FIG. 6. Nonlinear wave interactions among Kelvin (solid line), Yanai (dashed line) and first Poincaré (dotted line) modes. Two in-phase waves exist in the initial condition: $K=0.5 \cos (4 \theta), Y=0.15 \cos (4 \theta)$ and $P_{1}=0$, which are shown in (a). Snapshots are taken at (b) $\tau=2$ and (c) $\tau=5$. 
(a) $\tau=0$

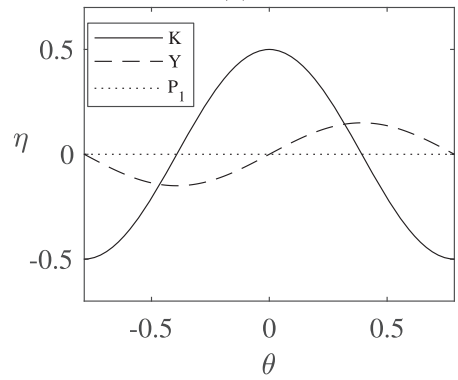

(b) $\tau=2$

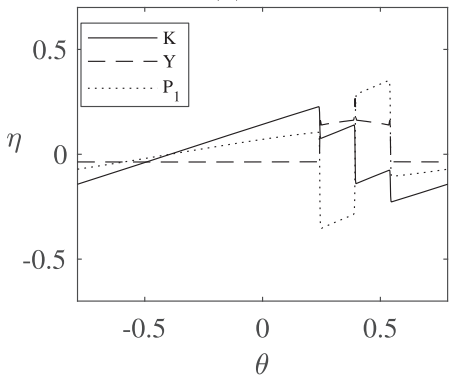

(c) $\tau=5$

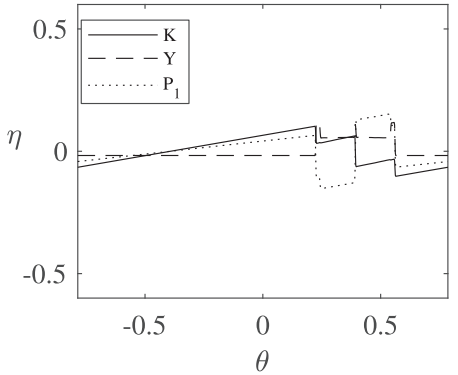

FIG. 7. Nonlinear wave interactions among Kelvin (solid line), Yanai (dashed line) and first Poincaré (dotted line) modes. Two out-of-phase waves exist in the initial condition: $K=0.5 \cos (4 \theta), Y=0.15 \sin (4 \theta)$ and $P_{1}=0$, which are shown in (a). Snapshots are taken at (b) $\tau=2$ and (c) $\tau=5$.

(a) $\tau=0$

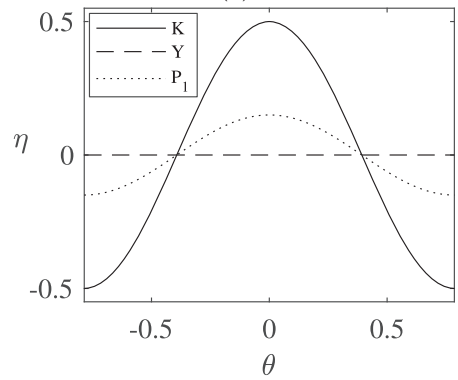

(b) $\tau=2$

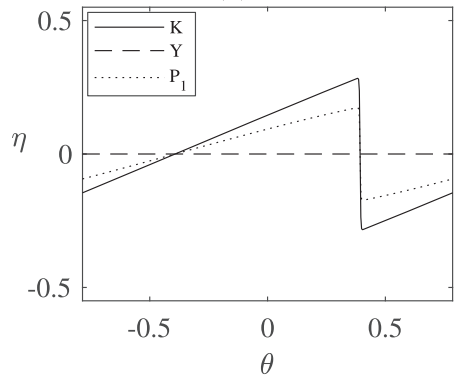

(c) $\tau=5$

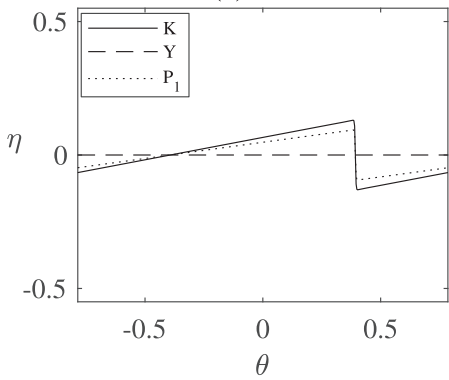

FIG. 8. Nonlinear wave interactions among Kelvin (solid line), Yanai (dashed line) and first Poincaré (dotted line) modes. Two in-phase waves exist in the initial condition: $K=0.5 \cos (4 \theta), Y=0$ and $P_{1}=0.15 \cos (4 \theta)$, which are shown in (a). Snapshots are taken at (b) $\tau=2$ and (c) $\tau=5$.

(a) $\tau=0$

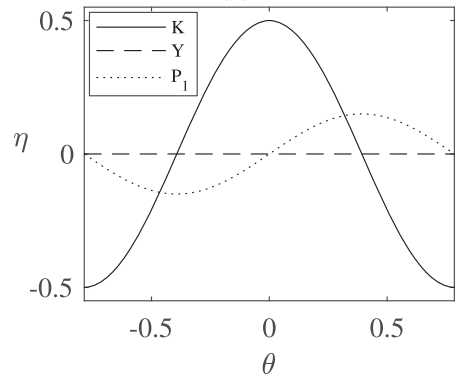

(b) $\tau=2$

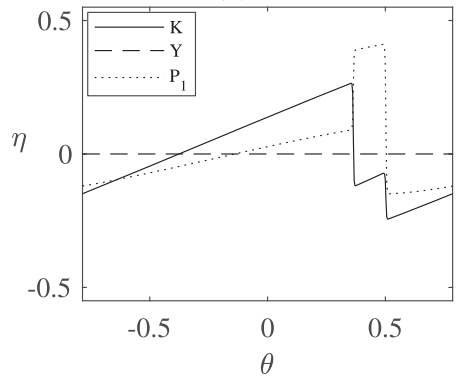

(c) $\tau=5$

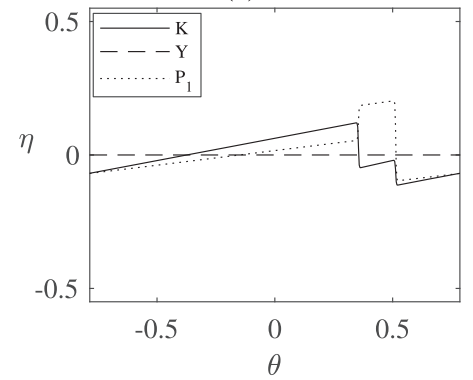

FIG. 9. Nonlinear wave interactions among Kelvin (solid line), Yanai (dashed line) and first Poincaré (dotted line) modes. Two out-of-phase waves exist in the initial condition: $K=0.5 \cos (4 \theta), Y=0$ and $P_{1}=0.15 \sin (4 \theta)$, which are shown in (a). Snapshots are taken at (b) $\tau=2$ and (c) $\tau=5$. 
initial condition, namely $K(\theta, 0)+Y(\theta, 0)=0$, similar phenomenon can be observed (though it is not shown here). These results further verified the properties deduced from (2.11).

Figures 6- 7 show the time evolution of the initial data consisting of a large-amplitude Kelvin wave and a small-amplitude Yanai wave. As shown in Fig. 6, three waves keep in phase during the whole process and shocks appear in the early stage of development. The Yanai wave decreases rapidly and almost disappears at $\tau=2$. The out-of-phase case is shown in Fig. 7, which is found to be more complicated than the in-phase case. In the beginning, the Kelvin wave pumps energy into the Yanai and Poincaré waves. The energy of the Yanai wave shows an increasing trend and peaks at $\tau=1$. Then, amplitudes of all three waves decrease due to shocks which dissipate energy in a periodic domain. It is noticed that the Yanai wave features a symmetrical structure at all times. We also carried out numerical experiments for a larger-amplitude Yanai wave initially and found that waves decrease more slowly.

Time evolutions of the initial combination of a large-amplitude Kelvin wave and a small-amplitude Poincaré wave are shown in Figs. 8 and 9 for in-phase and out-of-phase, respectively. The fundamental features of two examples are similar. No Yanai waves were found to appear in both cases. It can be understood by considering the odd/even nature of the modes, which indicates that terms like $\left(K^{2}\right)_{\theta}$, $\left(P_{1}^{2}\right)_{\theta}$ and $\left(K P_{1}\right)_{\theta}$ vanish in deriving the governing equation for the Yanai mode. The Kelvin wave transfers energy to the Poincaré wave until they reach a similar amplitude while the energy transfer is stronger in the out-of-phase case at the early stage. The phase property (in-phase or out-of-phase) is preserved during the whole process.

\subsection{Interaction among Kelvin, Yanai and second Poincaré modes}

In the previous subsection, the reduced model reveals that the characteristics of wave interactions among Kelvin, Yanai and first Poincaré modes depend on the initial phase relation and relative strength, while Yanai waves cannot be generated by other waves. To further study nonlinear interactions of different equatorial waves and the generation of Yanai waves, we propose another model including Kelvin, Yanai and second Poincaré modes. Based on the aforementioned arguments, the solution ansatz can be expressed as

$$
\begin{aligned}
& \eta=\epsilon e^{-y^{2} / 2}\left[K+y Y+\left(y^{3}-\frac{3}{2} y\right) P_{2}\right](\theta, \tau)+\epsilon^{2} \eta_{2}+\cdots, \\
& u=\epsilon e^{-y^{2} / 2}\left[K+y Y+\left(y^{3}-\frac{3}{2} y\right) P_{2}\right](\theta, \tau)+\epsilon^{2} u_{2}+\cdots, \\
& v=\epsilon e^{-y^{2} / 2}\left[Z+\left(y^{2}-\frac{1}{2}\right) Q_{2}\right](\theta, \tau)+\epsilon e^{-y^{2} / 2}\left[C_{3}+\left(y^{2}-\frac{1}{2}\right) C_{4}\right](x, \tau)+\epsilon^{2} v_{2}+\cdots,
\end{aligned}
$$

with

$$
Z_{\theta}=Y, \quad\left(Q_{2}\right)_{\theta}=3 P_{2},
$$

where $C_{i}(i=3,4)$ are arbitrary functions. The derivation of the governing equations for $K, Y$ and $P_{2}$ is similar to the previous subsection. Therefore, we state the result as follows without details:

$$
\left\{\begin{array}{c}
K_{\tau}+\sqrt{\frac{2}{3}}\left(\frac{3 K^{2}}{4}+\frac{Y^{2}}{4}-\frac{Y P_{2}}{4}+\frac{11 P_{2}^{2}}{48}\right)_{\theta}=0, \\
Y_{\tau}+\sqrt{\frac{2}{3}}\left(K Y-\frac{K P_{2}}{2}\right)_{\theta}=0, \\
\left(P_{2}\right)_{\tau}+\sqrt{\frac{2}{3}}\left(-\frac{K Y}{3}+\frac{11 K P_{2}}{18}\right)_{\theta}=0 .
\end{array}\right.
$$


(a) $\tau=0$

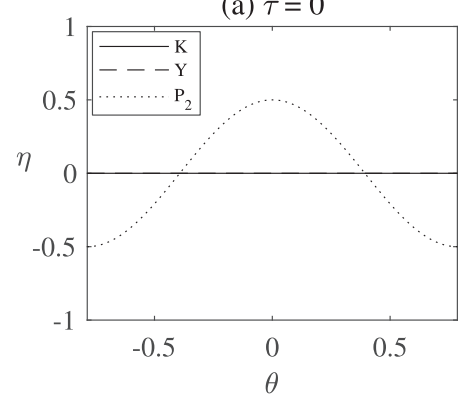

(b) $\tau=2$

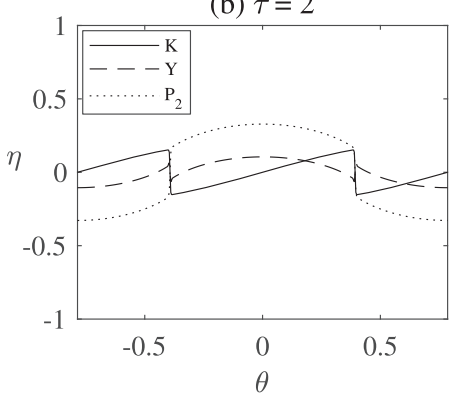

(c) $\tau=5$

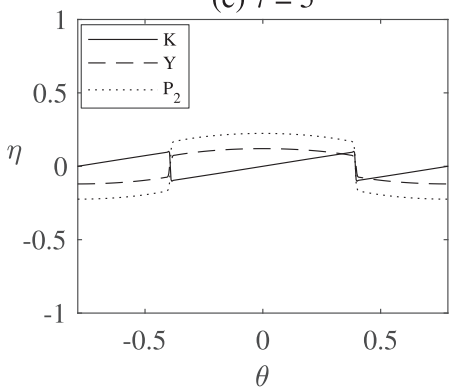

FIG. 10. Nonlinear wave interactions among Kelvin (solid line), Yanai (dashed line) and second Poincaré (dotted line) modes. A single Poincaré wave can awake the other two, and the initial condition is shown in (a): $K=Y=0$ and $P_{2}=0.5 \cos (4 \theta)$. Snapshots are taken at (b) $\tau=2$ and (c) $\tau=5$.

(a) $\tau=0$

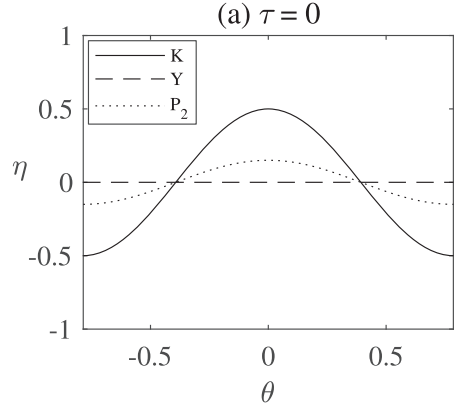

(b) $\tau=2$

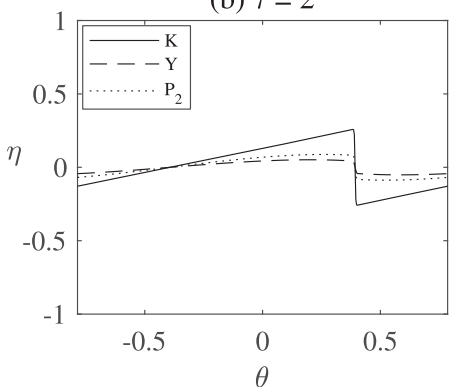

(c) $\tau=5$

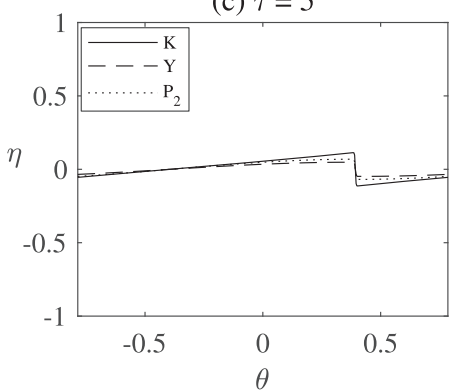

FIG. 11. Nonlinear interactions among Kelvin (solid line), Yanai (dashed line) and first Poincaré (dotted line) modes. Two in-phase waves are present in the initial condition: $K=0.5 \cos (4 \theta), Y=0$, and $P_{2}=0.15 \cos (4 \theta)$, which are shown in (a). Snapshots are taken at (b) $\tau=2$ and (c) $\tau=5$.

Next, we explore the excitation of Kelvin and Yanai waves by the second Poincaré wave based on numerical simulations for system (2.16). We assume no Kelvin and Yanai waves are initially present, and again the numerical solution to (2.16) was obtained using the fifth-order WENO scheme. We plot in Fig. 10 snapshots taken at different times. It is noticeable that the second Poincaré wave can produce both Kelvin and Yanai waves, and the generated Kelvin wave has half the wavelength of the other two.

Figures 11- 12 show the interaction dynamics and energy transfer when Kelvin and second Poincaré waves are initially present. Numerical experiments were carried out for different initial phase positions: in-phase (Fig. 11) and out-of-phase (Fig. 12). An interesting phenomenon is observed in the out-ofphase situation, where Kelvin and second Poincaré waves can produce a large-amplitude Yanai wave. In this case, amplitudes for both Kelvin and second Poincaré waves decrease while the Yanai wave shows an increasing trend at the early stage and peaks near $\tau=2$.

Models (2.11) and (2.16) provide us with a direct description of how these waves interact with each other and a new explanation for excitation of Yanai waves: even Poincaré modes can excite Kelvin waves through self-interaction and simultaneously excite Yanai waves via its interaction with the generated Kelvin waves. Multi-scale analysis and Galerkin projection help transform the primitive shallow water equations with the Coriolis force as source terms to a simple quasi-linear hyperbolic system of conservation laws. In addition, models can be easily simulated for long-time behaviours of waves due to the long-term parameter $\tau$, which helps reduce the calculation cost dramatically. 
(a) $\tau=0$

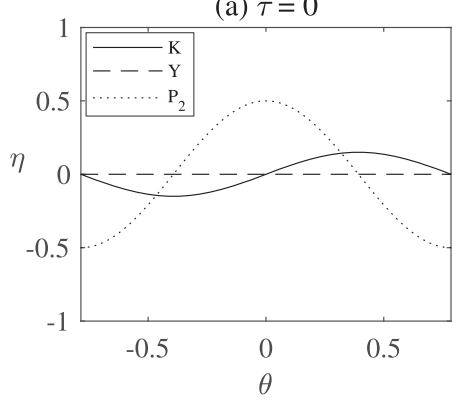

(b) $\tau=2$

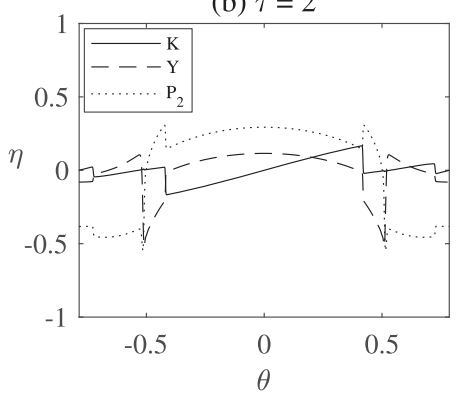

(c) $\tau=5$

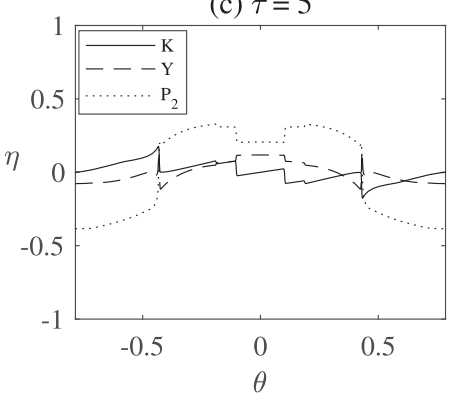

FIG. 12. Nonlinear wave interactions among Kelvin (solid line), Yanai (dashed line) and first Poincaré (dotted line) modes. Two out-of-phase waves are present in the initial condition: $K=0.15 \sin (4 \theta), Y=0$ and $P_{2}=0.5 \cos (4 \theta)$, which are shown in (a). Snapshots are taken at (b) $\tau=2$ and (c) $\tau=5$.

In the end, we list the model to include $K, Y, P_{1}$ and $P_{2}$ (i.e. four wave interactions), which is governed by the following equations:

$$
\left\{\begin{array}{c}
K_{\tau}+\sqrt{\frac{2}{3}}\left(-\frac{K P_{1}}{4}-\frac{Y P_{2}}{4}+\frac{3 K^{2}}{4}+\frac{Y^{2}}{4}+\frac{3 P_{1}^{2}}{16}+\frac{11 P_{2}^{2}}{48}\right)_{\theta}=0 \\
Y_{\tau}+\sqrt{\frac{2}{3}}\left(K Y-\frac{K P_{2}}{2}+\frac{Y P_{1}}{2}+\frac{5 P_{1} P_{2}}{12}\right)_{\theta}=0 \\
\left(P_{1}\right)_{\tau}+\sqrt{\frac{2}{3}}\left(\frac{3 K P_{1}}{4}+\frac{5 Y P_{2}}{12}-\frac{K^{2}}{4}+\frac{Y^{2}}{4}+\frac{13 P_{1}^{2}}{48}+\frac{49 P_{2}^{2}}{144}\right)_{\theta}=0 \\
\left(P_{2}\right)_{\tau}+\sqrt{\frac{2}{3}}\left(-\frac{K Y}{3}+\frac{11 K P_{2}}{18}+\frac{5 Y P_{1}}{18}+\frac{49 P_{1} P_{2}}{108}\right)_{\theta}=0 .
\end{array}\right.
$$

System (2.17) will be simulated and numerical results will be compare with the primitive equations in the next section.

We remark that following the same procedure the hyperbolic system can be generalized to include all Poincaré modes, and the derivation is sketched as follows. First of all, we make the following ansatz for the solution

$$
\left\{\begin{array}{l}
\eta=\varepsilon e^{-y^{2} / 2} \sum_{n=-1}^{N} H_{n+1}(y) P_{n}(\theta, \tau)+\varepsilon^{2} \eta_{2}+\cdots \\
u=\varepsilon e^{-y^{2} / 2} \sum_{n=-1}^{N} H_{n+1}(y) P_{n}(\theta, \tau)+\varepsilon^{2} u_{2}+\cdots \\
v=\varepsilon e^{-y^{2} / 2} \sum_{n=0}^{N} H_{n}(y) Q_{n}(\theta, \tau)+\varepsilon e^{-y^{2} / 2} \sum_{n=0}^{N} H_{n}(y) C_{n}(x, \tau)+\varepsilon^{2} v_{2}+\cdots
\end{array}\right.
$$

where we simply denote $K=P_{-1}, Y=P_{0}, H_{n}$ is the $n$-order monic Hermite polynomial and $\left(Q_{n}\right)_{\theta}=$ $(n+1) P_{n}$. Substituting the ansatz into (2.7) and collecting terms of $O\left(\varepsilon^{2}\right)$, one obtains

$$
\left(\eta_{2}\right)_{t}+\left(u_{2}\right)_{x}=-\sum_{n=-1}^{N} H_{n+1}(y)\left(P_{n}\right)_{\theta} e^{-y^{2} / 2}-\partial_{\theta}\left[\sum_{n=-1}^{N} H_{n+1}(y) P_{n}\right]^{2} e^{-y^{2}},
$$




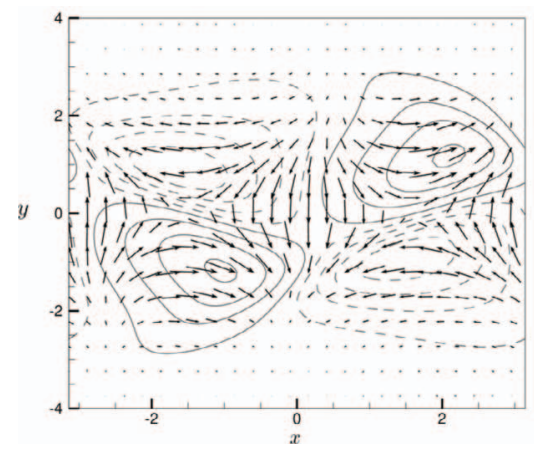

(a) $t=3$

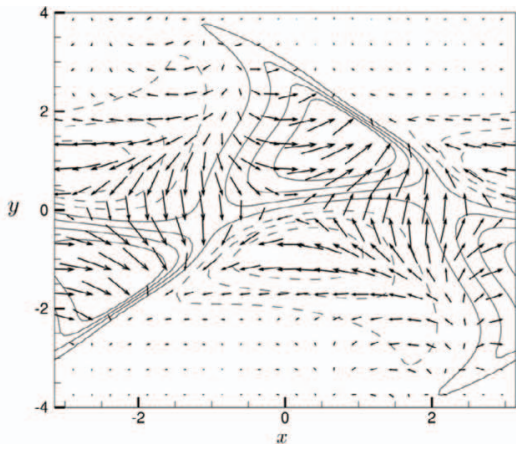

(b) $t=6$

FIG. 13. Direct numerical simulation for the primitive equations with the initial data given as $\eta_{0}=u_{0}=\epsilon e^{-\frac{y^{2}}{2}}(0.5 y \cos (x))$ and $v_{0}=\epsilon e^{-\frac{y^{2}}{2}}(0.5 \sin (x))$, where $\epsilon=0.05$. Snapshots of contour pictures are shown at (a) $t=3.0$ and (b) $t=6.0$.

$$
\left(u_{2}\right)_{t}+\left(\eta_{2}\right)_{x}=-\sum_{n=-1}^{N} H_{n+1}(y)\left(P_{n}\right)_{\tau} e^{-y^{2} / 2}-\frac{1}{2} \partial_{\theta}\left[\sum_{n=-1}^{N} H_{n+1}(y) P_{n}\right]^{2} e^{-y^{2}}
$$

The solvability conditions imply that the projection of the function

$$
\sum_{n=-1}^{N} H_{n+1}(y)\left(P_{n}\right)_{\tau} e^{-y^{2}}+\frac{3}{4} \partial_{\theta}\left[\sum_{n=-1}^{N} H_{n+1}(y) P_{n}\right]^{2} e^{-3 y^{2} / 2}
$$

onto arbitrary Hermite polynomial vanishes. Finally, the orthogonality of the Hermite polynomials indicates that the projection onto $H_{j+1}(y)$ gives the time-evolution equation for $P_{j}$.

\section{Solutions to the primitive equations}

In order to check whether the simplified models from the previous section are sufficient to study the interaction of nonlinear waves, we compare their solutions to a more sophisticated model and advanced numerical method. To this end, we solve the primitive equations and study the long-time behaviour of different equatorial waves and their interactions. The code used to perform this study is based on the MATLAB/GNU Octave toolbox FESTUNG (Finite Element Simulation Toolbox for UNstructured Grids), and the interested reader is referred to (Frank et al., 2015; Jaust et al., 2018; Reuter et al., 2016) for the complete description of this alogerithm. We solve the shallow water equations with the discontinuous Galerkin method and strong stability preserving (SSP) Runge-Kutta time integration with second-order accuracy in space and time. The primitive equations can be rewritten in a compact form as

$$
\mathbf{U}_{t}+\nabla \cdot \mathbf{F}(\mathbf{U})=\mathbf{S}(\mathbf{U})
$$




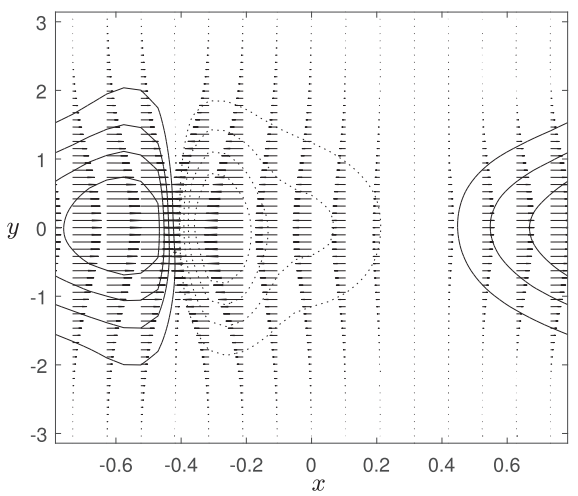

(a) Result of the primitive equations at $t=40$

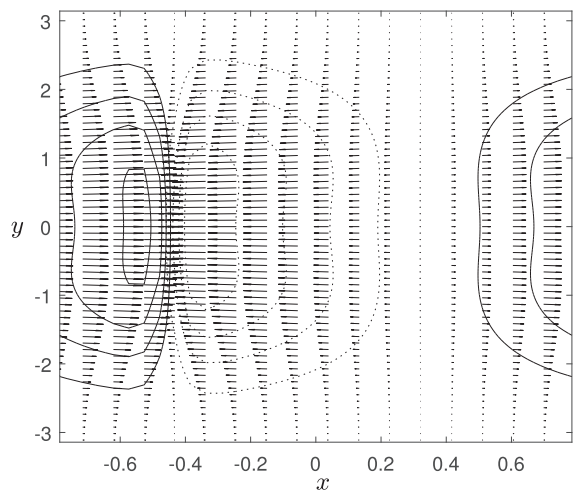

(b) Result of the model of four waves at $\tau=2$

FIG. 14. Comparison between the primitive equations and the four-mode model. The initial condition is a single Kelvin wave: $\eta_{0}=u_{0}=\epsilon e^{-\frac{y^{2}}{2}}(0.5 \cos (4 x))$ and $v_{0}=0$.

where

$\mathbf{U}=\left[\begin{array}{c}\eta \\ (H+\eta) u \\ (H+\eta) v\end{array}\right], \mathbf{S}(\mathbf{U})=\left[\begin{array}{c}0 \\ \beta y(H+\eta) v \\ -\beta y(H+\eta) u\end{array}\right], \mathbf{F}(\mathbf{U})=(H+\eta)\left[\begin{array}{cc}u & v \\ u^{2}+\frac{g}{2}(H+\eta) & u v \\ v u & v^{2}+\frac{g}{2}(H+\eta)\end{array}\right]$

The velocity is found from an interpolation of the quotient of momentum and water height. The following element local variational formulation is derived by testing (3.1) with a test function $\mathbf{w}_{h}$, i.e. zero outside of an element $K^{-}$of the computational mesh:

$$
\int_{K^{-}} \mathbf{w}_{h} \cdot \partial_{t} \mathbf{U}_{h} \mathrm{~d} \mathbf{x}-\int_{K^{-}} \nabla \mathbf{w}_{h}: \mathbf{F}\left(\mathbf{U}_{h}\right) \mathrm{d} \mathbf{x}+\int_{\partial K^{-}} \mathbf{w}_{h} \cdot \widehat{\mathbf{F}}\left(\mathbf{U}_{h}^{-}, \mathbf{U}_{h}^{+} ; \mathbf{n}_{K^{-}}\right) \mathrm{d} s=\int_{K^{-}} \mathbf{w}_{h} \cdot \mathbf{S}\left(\mathbf{U}_{h}\right) \mathrm{d} \mathbf{x} .
$$

Here, $\mathbf{n}_{K^{-}}$is the unit outward normal vector and $\widehat{\mathbf{F}}\left(\mathbf{U}_{h}^{-}, \mathbf{U}_{h}^{+} ; \mathbf{n}_{K^{-}}\right)$is a numerical flux defined in terms of the one-sided limits $\mathbf{U}_{h}^{ \pm}$, which belong to either $K^{-}$or the appropriate neighbouring element $K^{+}$. A full description of the employed numerical methods can be found in Liu et al. (1994).

There are many advantages to using this model over the simplified ones discussed above: it describes the $2 \mathrm{D}$ shallow water physics to full extend, the conservative form of equation (3.1) assures convergence to the entropy solution and the discontinuous finite element approximation guarantees local conservation of mass and momentum.

First, we study the behaviour for the case where only a Yanai wave is present initially (Fig. 13). The results are consistent with Bouchut's second-order, well-balanced finite volume method (Bouchut et al., 2005), which verifies our approach.

Next, we consider a square domain with a periodic boundary condition in the $x$ direction and radiation condition in the $y$ direction and compare the model equations solved with a fifth-order WENO method (Liu et al., 1994) and SSP Runge-Kutta time discretization to the primitive solution. The variable $\tau=\epsilon t$ is used for the time scale of the model equations; in this section, we set $\epsilon=0.05$. 


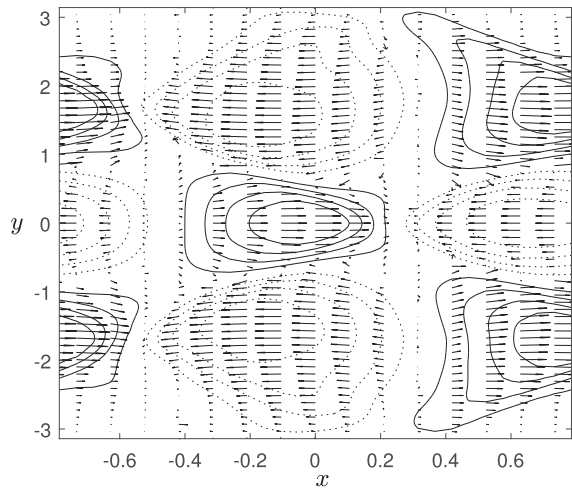

(a) Result of the primitive equations at $t=40$

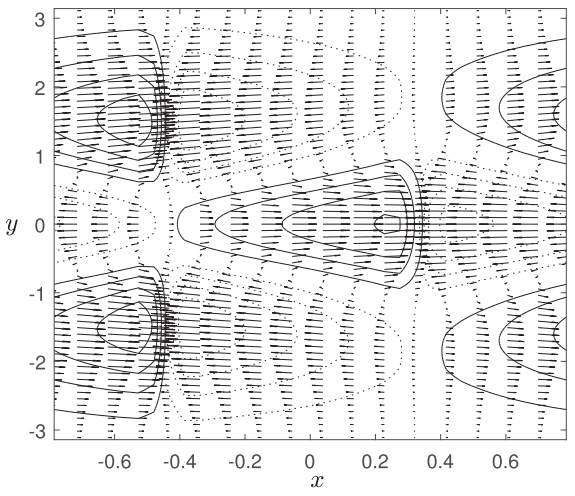

(b) Result of the model of four waves at $\tau=2$

FIG. 15. Comparison between the primitive equations and the four-mode model. The initial condition only contains a first Poincaré mode: (a) $\eta_{0}=u_{0}=\epsilon e^{-\frac{y^{2}}{2}}(0.5 \cos (4 x)+0.15 y \cos (4 x))$ and $v_{0}=\epsilon e^{-\frac{y^{2}}{2}}(0.0375 \sin (4 x)) ;(\mathrm{b}) \eta_{0}=u_{0}=$ $\epsilon e^{-\frac{y^{2}}{2}}\left[0.15 \cos (4 x)+0.5\left(y^{3}-\frac{3}{2} y\right) \cos (4 x)\right]$ and $v_{0}=\epsilon e^{-\frac{y^{2}}{2}}\left[0.375\left(y^{2}-\frac{1}{2}\right) \sin (4 x)\right] ;(\mathrm{c}) \eta_{0}=u_{0}=\epsilon e^{-\frac{y^{2}}{2}}(0.5 \cos (4 x)+$ $0.15 y \sin (4 x))$ and $v_{0}=\epsilon e^{-\frac{y^{2}}{2}}(-0.0375 \cos (4 x)) ;(\mathrm{d}) \eta_{0}=u_{0}=\epsilon e^{-\frac{y^{2}}{2}}\left[0.15 \sin (4 x)+0.5\left(y^{3}-\frac{3}{2} y\right) \cos (4 x)\right]$ and $v_{0}=$ $\epsilon e^{-\frac{y^{2}}{2}}\left[0.375\left(y^{2}-\frac{1}{2}\right) \sin (4 x)\right]$. The centreline snapshot is shown at $t=40$ (equivalent to $\tau=2$ ).

In Figs. 14- 16, we compare the results of the primitive equations at time $t=40$ to the model equations at $\tau=2$ for different initial conditions. A Dirichlet zero boundary condition is imposed in the $y$ direction owing to the trapped nature of ESW waves, and obviously waves are periodic in the $\theta$ direction. It is noted that numerical experiments for the primitive equations with periodic boundary conditions in both zonal and meridional directions have been carried out for comparison purposes, which show good agreement with results from the zero boundary condition in the meridional direction. Three models containing different modes have been listed in the previous section. Since the four-mode model is compatible with the other two three-mode models, we only show the configuration of the four-mode model (2.17). In general, we observe similarity in wave profiles, at least in the qualitative sense, between the reduce model and primitive solutions.

Figures 14 and 15 show respectively the solutions with only Kelvin and first Poincaré waves present initially. We observe that in both cases, the free surface elevation is almost symmetric about the $x$-axis, so we can conclude that there are no Yanai and seco

nd Poincare waves at any point of the simulation, which is consistent with the asymptotic analysis of the previous section. Shocks can be observed both in the simulation of the primitive equations and model equations. When there is only Kelvin wave initially, the positions of shocks and the velocity fields derived from the primitive equations are close to that of the model equations. When there is only the first Poincaré wave initially, there are some differences in the detailed structures, although the general characteristics are consistent with each other.

When the initial condition is posed by the combination of a large-amplitude Kelvin wave and an inphase Yanai wave, it is demonstrated in Fig. 16(a) that shock waves form both in the primitive equations and in the model equations and locations of the shocks show a remarkable agreement. Figure 16(b) shows the development of small-amplitude Kelvin and in-phase Poincaré wave. Figures 16(c and d) 
(a)

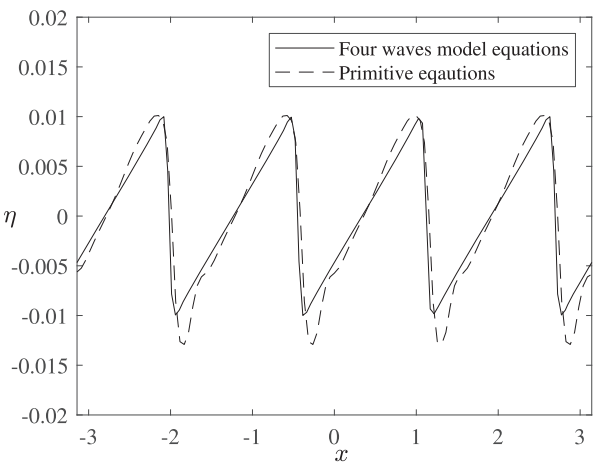

(b)
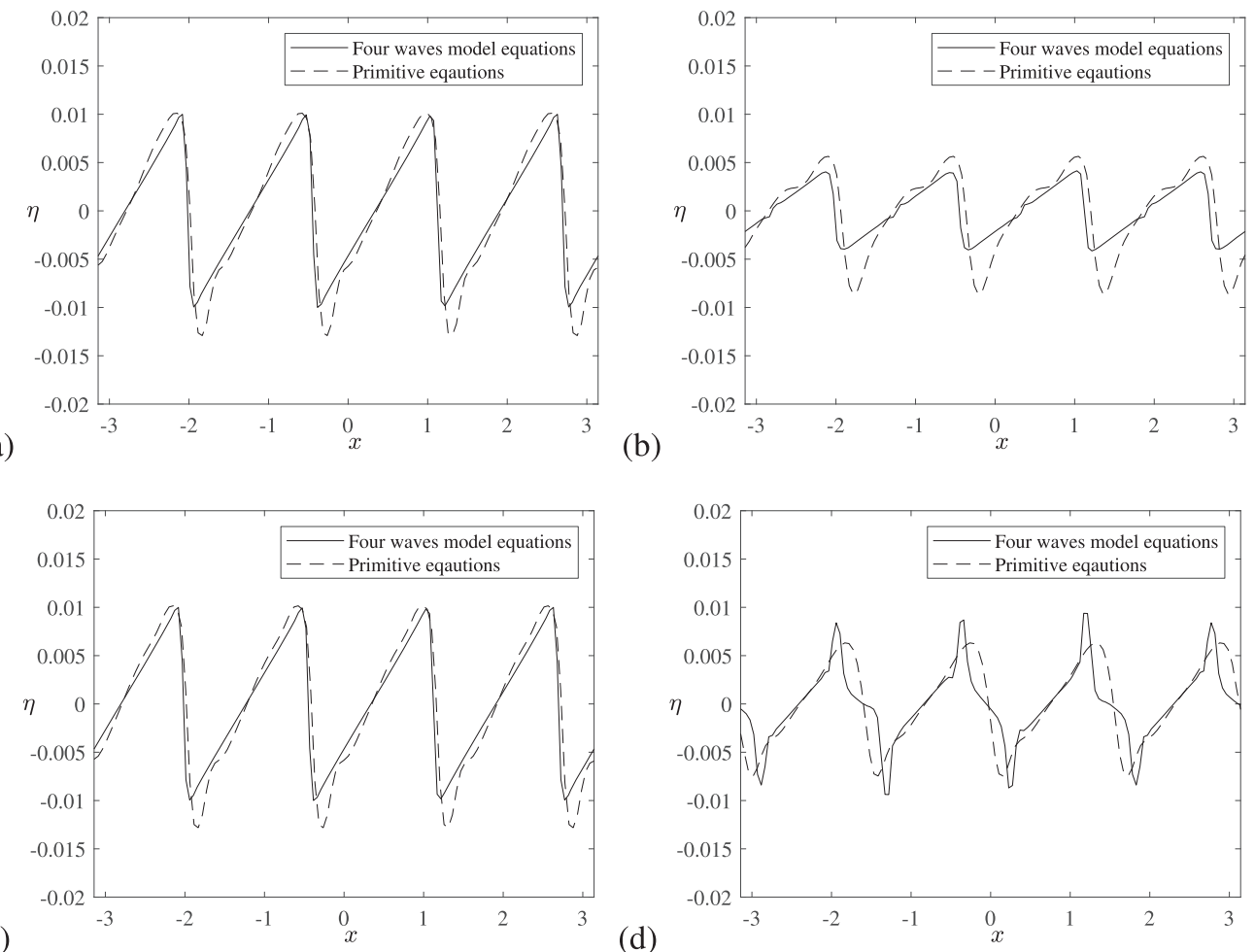

(d)

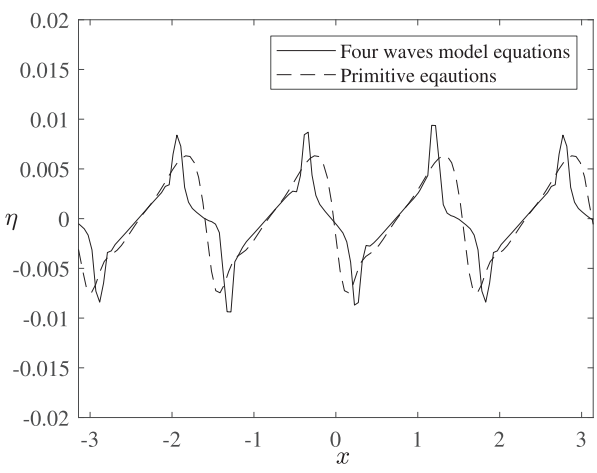

FIG. 16. Comparison between the primitive equations and the four-mode model. The initial condition only contains a first Poincaré mode: $\eta_{0}=u_{0}=\epsilon e^{-\frac{y^{2}}{2}}\left(0.5\left(y^{2}-\frac{1}{2}\right) \cos (4 x)\right)$ and $v_{0}=\epsilon e^{-\frac{y^{2}}{2}}(0.25 y \sin (4 x))$.

show the out-of-phase cases where Kelvin and Yanai modes and Kelvin and Poincaré modes are present initially. It is shown that the model captures shocks exactly; however, the results are somewhat different in fine structures presumably due to the lack of dispersive effects in reduced models.

\section{Concluding remarks}

A lot of field observations have found wavelike disturbances with a period of the order of 4 and 5 days and zonal wavenumbers $4-5$, with a structure very close to the Kelvin and eastward-propagating Yanai waves, indicating that these waves constitute a prominent characteristic of the tropical atmospheric circulation. As shown in Fig. 1, the phase speed of different linear modes are quite close when wavenumbers are greater than 3; therefore, they are more likely to interact with each other in the longtime dynamics where nonlinear effects become efficient. Based on these fundamental observations, we have presented three new models to describe nonlinear wave interactions among equatorial modes using a multi-scale method. It is found that the second Poincaré modes can excite Yanai waves, which is a new possible explanation for the strong spectral peak band in intermediate scale eastward-propagating Yanai waves observed in the tropical atmosphere. In addition, the Yanai mode or the second Poincaré mode alone can excite other types of waves and this conclusion can be generalized to other Poincaré modes for 
even $n$ in the dispersion relation (2.6). Furthermore, the characteristics of nonlinear wave interactions are found to be associated with types of initial mode, wave amplitudes and relative phase. Particularly, it is shown that initial modes with different phases are more likely to result in a strong energy transfer than the in-phase scenario.

We further solve scenarios with a more sophisticated method for the primitive shallow water equations with the Coriolis force. The comparison of results between models and the primitive equations indicates the validity of the simplified models. It is remarked that though not explicitly represented in figures, we also carried out numerical simulations for various values of $\delta(0 \leqslant \delta \leqslant 1)$ in system (2.7), the parameter accounting for short waves. The numerical results for zonal wavenumber $k=4$ show that the relative amplitude error between two different $\delta \mathrm{s}$ is less than $6 \%$ at $t=6$, partially confirming that the complete removal of the $\delta$-related terms is a reasonable approximation for the dynamics of short waves. For numerics, the advantages of simplified models are twofolds: dimensionality reduction and algorithmic simplification. In contrast to the primitive equations, the models are all quasi-linear hyperbolic systems of conservation laws, for which there exist a variety of established numerical methods. On physical grounds, simplified models can explain the mechanism of nonlinear wave interactions of intermediate-scale equatorial waves in an explicit manner.

We can draw a conclusion from the present work that nonlinear interactions of nearly non-dispersive, intermediate-scale equatorial waves may play a role in atmospheric motions. We point out that, while our methodology is valid to study many wave-interaction phenomena in the tropics, it is limited to configurations where one can omit certain effects. In practice, one needs to consider more effects such as solar energy, density stratification, bottom friction, etc. Nevertheless, as demonstrated in Section 3, our simplified models often capture essential features of interest in more complicated fluid mechanics. Finally, we remark that the asymptotic and numerical techniques presented in this paper can be generalized to other problems, such as equatorial magnetohydrodynamic shallow water equations (see, e.g. Zaqarashvili, 2018).

\section{Acknowledgements}

ZW wishes to thank Professor Paul Milewski (University of Bath) for his initial guidance to the problems of equatorial waves and helpful discussions.

\section{Funding}

DFG Initiation of International Collaboration Programme (KU 1530/22-1); Key Research Program of Frontier Sciences of CAS (QYZDBSSW-SYS015); Strategic Priority Research Program of the Chinese Academy of Sciences (XDB22040203); CAS Center for Excellence in Complex System Mechanics.

\section{REFERENCES}

Baldwin, M., Gray, L., Dunkerton, T., Hamilton, K., Haynes, P., Randel, W. J., Holton, J., Alexander, M., Hirota, I., Horinouchi, T., Jones, D., Kinnersley, J., Marquardt, C. \& Takahashi, K. S. M. (2001) The quasi-biennial oscillation. Rev. Geophys., 39, 179-229.

Bouchut, F., Le, S. \& Zeitlin, V. (2005) Breaking of balanced and unbalanced equatorial waves. Chaos, $15,25$. Boyd, J. (1980) The nonlinear equatorial kelvin wave. J. Phys. Oceanogr., 10, 1552-1573.

Boyd, J. (1983a) Long wave/short wave resonance in equatorial waves. J. Phys. Oceanogr., 13, 450-458. Boyd, J. (1983b) Second harmonic resonance for equatorial waves. J. Phys. Oceanogr., 13, 459-466. 
Frank, F., Reuter, B., Aizinger, V. \& Knabner, P. (2015) Festung: a MATLAB/GNU octave toolbox for the discontinuous galerkin method. Part I: diffusion operator. Comp. Math. Appl., 70, 11-46.

Iтон, H. \& GHIL, M. (1988) The generation mechanism of mixed rossby-gravity waves in the equatorial troposphere. J. Atmos. Sci., 45, 585-604.

Jaust, A., Reuter, B., Aizinger, V., SchÜtz, J. \& Knabner, P. (2018) Festung: a MATLAB/GNU octave toolbox for the discontinuous galerkin method. Part III: hybridized discontinuous galerkin (HDG) formulation. Comp. Math. Appl., 75, 4505-4533.

Kelly, B., Meyers, S. \& Brien, J. (1995) On a generating mechanism for Yanai waves and the 25-day oscillation. J. Geophys. Res. Oceans, 100, 10589-10612.

Kiladis, G., Wheeler, M., Patrick, P., Straub, K. \& Roundy, P. (2009) Convectively coupled equatorial waves. Rev. Geophys., 47, 25-42.

LinDZEN, R. (1967) Planetary waves on beta-planes. Mon. Weather Rev., 95, 441-451.

Liu, X.-D., Osher, S. \& Chan, T. (1994) Weighted essentially non-oscillatory schemes. J. Comput. Phys., 115, 200-212.

Majda, A., Rosales, R., TABAK, E. \& Turner, C. (1999) Interaction of large-scale equatorial waves and dispersion of kelvin waves through topographic resonances. J. Atmos. Sci., 56, 4118-4133.

Marcus, M. (2008) Resonant wave interactions in the equatorial waveguide. J. Atmos. Sci., 65, 3398-3418.

Maruyama, T. (1967) Large-scale disturbances in the equatorial lower stratosphere. J. Meteor. Soc. Japan, 45, 391-408.

Matsuno, T. (1966) Quasi-geostrophic motions in the equatorial area. J. Meteor. Soc. Japan, 44, 25-42.

RAUPP, C. \& DiAs, P. S. (2005) Excitation mechanism of mixed rossby-gravity waves in the equatorial atmosphere: role of the nonlinear interactions among equatorial waves. J. Atmos. Sci., 62, 1446-1462.

RaupP, C. \& DiAs, P. S. (2010) Dynamics of resonantly interacting equatorial waves. Tellus A, 58, 263-279.

Reuter, B., Aizinger, V., Wieland, M., Frank, F. \& Knabner, P. (2016) Festung: a MATLAB/GNU octave toolbox for the discontinuous galerkin method. Part II: advection operator and slope limiting. Comp. Math. Appl., 72, 1896-1925.

RIPA, P. (1982) Nonlinear wave wave interactions in a one-layer reduced-gravity model on the equatorial $\beta$ plane. J. Phys. Oceanogr., 12, 97-111.

RIPA, P. (1983a) Weak interactions of equatorial waves in a one-layer model. Part II. Applications. J. Phys. Oceanogr., 13, 1227-1240.

RIPA, P. (1983b) Weak interactions of equatorial waves in a one-layer model. Part I. General properties. J. Phys. Oceanogr., 13, 1208-1226.

TAKAYABU, Y. (1994) Large-scale cloud disturbances associated with equatorial waves. I: spectral features of the cloud disturbances. J. Meteorol. Soc. Japan., 3, 433-449.

Wallace, J. (1973) General circulation of the tropical lower stratosphere. Rev. Geophys., 11, 191-222.

WheELER, M. \& Kiladis, G. (1999) Convectively coupled equatorial waves: analysis of clouds and temperature in the wavenumber-frequency domain. J. Atmos. Sci., 56, 374-399.

YANAI, M. \& MARuYAma, T. (1966) Stratospheric wave disturbances propagating over the equatorial pacific. $J$. Meteor. Soc. Japan, 44, 291-294.

Yoshikazu, H. (1970) A theory of large-scale equatorial waves generated by condensation heat and accelerating. J. Meteor. Soc. Japan, 48, 140-160.

ZAQARASHVILI, T. (2018) Equatorial magnetohydrodynamic shallow water waves in the solar tachocline. Astrophys. $J ., 856,32$. 